\title{
CONCENTRAÇÃO DE PARTÍCULAS MINERAIS FINAS E ULTRAFINAS POR ELETROFLOTAÇÃO
}

\author{
E.P. Santos ${ }^{1}$, A.J.B. Dutra ${ }^{1}$ \\ 1 Programa de Engenharia Metalúrgica e de Materiais, COPPE/UFRJ \\ Cidade Universitária - Centro de Tecnologia - Rio de Janeiro, RJ, 21945-970 \\ everton.pedroza@metalmat.ufri.br - adutra@metalmat.ufri.br
}

Artigo submetido em novembro/2011 e aceito em dezembro/2011

\section{RESUMO}

A baixa recuperação na flotação de partículas minerais finas e ultrafinas continua sendo um dos principais desafios da área de processamento mineral. As técnicas emergentes existentes para o aumento da recuperação destas partículas são baseadas em novos conceitos de otimização da "captura" de partículas por bolhas, através do aumento da probabilidade de colisão bolhapartícula, injetando bolhas médias $(100-600 \mu \mathrm{m})$ e pequenas $(<100 \mu \mathrm{m})$ na célula de flotação. Dentre as técnicas existentes para a geração de bolhas pequenas, estão a flotação por ar dissolvido (FAD) e a eletroflotação (EF). Na EF são empregadas microbolhas de oxigênio e hidrogênio, geradas a partir da decomposição eletrolítica da água. Estas bolhas, com diâmetros variando entre 20 a $50 \mu \mathrm{m}$, são menores que as obtidas na FAD. As unidades de EF são pequenas e compactas e apresentam baixos custos de manutenção e funcionamento, quando comparadas a outros processos de flotação. Diversos estudos têm demonstrado a viabilidade do processo de EF aplicado ao tratamento de águas e de efluentes industriais, porém poucos estudos abordam a utilização desta técnica na área de processamento mineral. Este trabalho apresenta uma revisão da literatura sobre a utilização da EF aplicada ao tratamento de partículas minerais finas e ultrafinas.

PALAVRAS-CHAVE: finos, ultrafinos, microbolhas, eletroflotação

\section{CONCENTRATION OF FINE AND ULTRAFINE MINERAL PARTICLES BY ELECTROFLOTATION}

\section{ABSTRACT}

The low recovery in the flotation of fine and ultrafine mineral particles is still a challenge to be overcome in the area of mineral processing. Presently, the emerging techniques for increasing the recovery of such particles are based on new concepts of optimization of the "capture" of particles by bubbles through the increase of the probability of bubble-particle collision, which can be achieved by the injection of medium $(100-600 \mu \mathrm{m})$ or small $(<100 \mu \mathrm{m})$ bubbles in the flotation cell. Among the existing techniques for the generation of small bubbles, are dissolved air flotation (DAF) and electroflotation (EF). EF uses oxygen and hydrogen microbubbles generated by electrolytic decomposition of water, with a diameter ranging from 20 to $50 \mu \mathrm{m}$, values smaller than those obtained in FAD. EF units are small and compact and present low maintenance and operating costs when compared to other processes of flotation. Several studies have demonstrated the feasibility of the EF process applied to water and industrial effluent treatments, but only in few studies this technique is applied in the area of mineral processing. This paper presents a literature review about the use of EF applied to the treatment of fine and ultrafine mineral particles.

KEY-WORDS: fine, ultrafine, microbubbles, electroflotation 


\section{CONCENTRAÇÃO DE PARTÍCULAS MINERAIS FINAS E ULTRAFINAS POR ELETROFLOTAÇÃO}

\section{INTRODUÇÃO}

O esgotamento progressivo dos depósitos minerais aliado ao aumento da preocupação com a conservação dos recursos não renováveis tem forçado o setor mineral a tratar minérios de baixos teores e finamente disseminados. Essa disseminação requer uma moagem excessiva para liberação dos minerais, o que torna possível a separação seletiva, levando a geração e, conseqüentemente, a necessidade de processamento de partículas finas e ultrafinas.

A baixa recuperação na flotação de partículas minerais finas e ultrafinas continua sendo um dos principais desafios da área de processamento mineral (Trahar, 1981). Diversos autores (Collins e Read, 1971; Trahar e Warren, 1976; Trahar, 1981; Sivamohan, 1990; Rubio et al., 2003) têm descrito, detalhadamente, as dificuldades existentes em relação ao processo de flotação. A flotação convencional das partículas finas e ultrafinas juntamente com as partículas grossas é ineficiente devido a fenômenos que derivam de suas características intrínsecas, tais como: pequena massa, alta superfície específica e alta energia superficial.

Os principais problemas observados na flotação dessas frações são: baixa probabilidade de colisão e adesão entre partículas e bolhas, dificuldade de superar a barreira energética entre elas, arraste mecânico, recobrimento por ultrafinos de ganga (slime coating), elevado consumo de reagentes, rigidez excessiva da espuma, e baixa seletividade na adsorção de reagentes, além de aspectos como composição e oxidação superficial das partículas, alterações mineralógicas e íons dissolvidos na água de processo (Song et al., 2001). Todos esses fatores contribuem para uma diminuição da velocidade (cinética) de flotação e da recuperação do mineral de interesse, além de um aumento no consumo de reagentes. Por outro lado, as partículas grossas apresentam o problema contrário devido à sua massa elevada e baixo grau de liberação, o que leva a uma baixa recuperação.

Normalmente as partículas ultrafinas, com tamanho menor que $10 \mu \mathrm{m}$, têm baixa eficiência de colisão com bolhas de ar e são susceptíveis ao mecanismo de arraste hidrodinâmico. Este mecanismo não distingue as partículas hidrofílicas das hidrofóbicas e está estritamente relacionado com a recuperação de água. Em alguns trabalhos (Trahar, 1981; Warren, 1985; Subrahmanyam e Forssberg, 1990) observou-se uma relação linear entre a recuperação de água e do mineral, introduzindo os conceitos de grau de arraste hidrodinâmico e recuperação real, que é a obtida somente através do mecanismo bolhapartícula.

De acordo com Rubio et al. (2004) em qualquer sistema de flotação existe uma determinada faixa de tamanho de partículas para a qual deve-se dispor de uma distribuição de tamanho de bolhas adequada para que a "captura" de partículas pelas bolhas seja maximizada. Para uma ampla distribuição granulométrica de partículas, teoricamente necessita-se de uma distribuição de tamanho de bolhas correspondente. Isto não ocorre nas condições atuais de flotação em usinas de tratamento de minérios e por isso, a recuperação das partículas minerais finas e ultrafinas é normalmente baixa. 
As células de flotação convencionais geram bolhas com tamanho da ordem de 600 a $2000 \mu \mathrm{m}$. Alguns difusores podem gerar bolhas de tamanho médio (100 a $600 \mu \mathrm{m}$ ), porém ainda insuficiente para a flotação de partículas ultrafinas (Yoon, 1993).

As técnicas emergentes existentes para o aumento da recuperação de finos e ultrafinos são baseadas em novos conceitos de otimização da "captura" de partículas por bolhas, através do aumento da probabilidade de colisão bolha-partícula pelo aumento da distribuição de tamanho de bolhas na célula, com injeção de bolhas médias (100-600 $\mu \mathrm{m})$ ou pequenas (< $100 \mu \mathrm{m}$ ) (Song et al., 2001; Rubio et al., 2003).

Muitos estudos tem reportado que a recuperação de partículas de diâmetro entre 1 e $10 \mu \mathrm{m}$ tem aumentado com a diminuição do tamanho de bolhas, já que ocorre um aumento significativo da eficiência de colisão entre as bolhas e as partículas (Nguyen et al., 2006; Sarrot et al., 2007).

Técnicas alternativas têm sido desenvolvidas para a geração de bolhas finas e ultrafinas, como as técnicas de flotação por ar dissolvido (FAD) e eletroflotação (Ketkar et al., 1991; Burns et al., 1997).

\section{TAMANHO DAS BOLHAS GERADAS NO PROCESSO DE ELETROFLOTAÇÃO}

A eletroflotação emprega microbolhas de oxigênio e hidrogênio geradas a partir da decomposição eletrolítica da água. Estas microbolhas são mais homogêneas e apresentam uma dispersão mais uniforme na célula de flotação quando comparadas com as bolhas geradas pelo processo convencional (Venkatachalam et al., 1992). Estes autores ainda afirmam que no processo de eletroflotação, o diâmetro das bolhas formadas pode variar de 20 a $50 \mu \mathrm{m}$, valores abaixo dos obtidos na flotação por ar dissolvido e também na flotação convencional (Venkatachalam et al., 1992; Burns et al., 1997). A influência da densidade de corrente na geração de bolhas é muito importante no processo de separação de partículas, pois o aumento da densidade de corrente ocasionará uma maior liberação de gás. Quanto à variação no tamanho das bolhas em diferentes densidades de corrente, existem estudos conflitantes na literatura (Venkatachalam et al., 1992).

Diversos autores verificaram que o tamanho das bolhas produzidas por eletroflotação é influenciado por uma série de fatores, tais como: densidade da corrente, material dos eletrodos, tipo e concentração do eletrólito suporte e pH da polpa (Glembotskii et al., 1973; Ketkar et al., 1991).

Estudos reportam que o aumento da densidade de corrente provocou um aumento do tamanho das bolhas de hidrogênio, possivelmente pela coalescência dessas bolhas (Janssen e Hoogland, 1970; Landolt et al., 1970; Ben Mansour et al., 2007; Sarkar et al., 2010). A Tabela 1 apresenta o diâmetro médio das bolhas de hidrogênio e oxigênio, obtidos por Inan et al. (2004), em função da densidade de corrente. 
Tabela 1. Diâmetro médio das bolhas de hidrogênio e oxigênio variando a densidade de corrente (adaptada de Inan et al., 2004).

\begin{tabular}{c|c|c}
\hline Densidade de corrente & Bolhas de hidrogênio & Bolhas de oxigênio \\
\hline $100 \mathrm{~A} \cdot \mathrm{m}^{-2}$ & $15 \mu \mathrm{m}$ & $45 \mu \mathrm{m}$ \\
\hline $400 \mathrm{~A} \cdot \mathrm{m}^{-2}$ & $30 \mu \mathrm{m}$ & $60 \mu \mathrm{m}$ \\
\hline
\end{tabular}

Por outro lado, Venczel (1970) apud Venkatachalam et al., (1992) afirmaram que o aumento da densidade de corrente provocou a diminuição do tamanho das bolhas. Estudos realizados por Ketkar et al. (1991), corroboram esta afirmação, onde foi observado que os diâmetros das bolhas de hidrogênio e oxigênio diminuíram com o aumento da densidade da corrente, utilizando diferentes materiais de eletrodos, o que está de acordo com a teoria da nucleação e crescimento de uma nova fase sobre um eletrodo (Walsh e Herron, 1991). A Tabela 2 apresenta os resultados obtidos por Ketkar et al. (1991).

Tabela 2. Diâmetro das bolhas de hidrogênio e oxigênio variando a densidade de corrente (adaptada de Ketkar et al., 1991).

\begin{tabular}{c|c|c}
\hline Densidade de corrente & Bolhas de hidrogênio & Bolhas de oxigênio \\
\hline $125 \mathrm{~A} \cdot \mathrm{m}^{-2}$ & 34 a $49 \mu \mathrm{m}$ & 48 a $50 \mu \mathrm{m}$ \\
\hline $375 \mathrm{~A} \cdot \mathrm{m}^{-2}$ & 22 a $37 \mu \mathrm{m}$ & 38 a $42 \mu \mathrm{m}$ \\
\hline
\end{tabular}

No caso do material dos eletrodos, Venkatachalam et al. (1992) afirmam que a influência do material do catodo no tamanho das bolhas de hidrogênio é extremamente pronunciada em meio ácido e menos acentuada em meio alcalino. Já em meio neutro, o material do catodo não interfere no tamanho das bolhas de hidrogênio. $O$ tamanho das bolhas geradas por eletroflotação é alterado com a mudança no material do eletrodo, como mostrado na Figura 1.

$$
\mathrm{Pb}, \stackrel{\mathrm{Sn}, \mathrm{Cu}, \mathrm{Ag}, \mathrm{Fe}, \mathrm{Ni}, \mathrm{W}, \mathrm{Pd}, \mathrm{Pt}}{\longrightarrow}
$$

Aumento do tamanho das bolhas de Oxigênio

Figura 1. Influência do tipo de material no tamanho das bolhas geradas eletroliticamente (adaptada de Venkatachalam et al., 1992).

Já para o caso do $\mathrm{pH}$ da polpa, estudos mostram que pequenas bolhas de hidrogênio são obtidas em pH neutro ou levemente alcalino, e que o tamanho das bolhas de oxigênio de menor diâmetro são obtidas em pH levemente ácido e crescem com o aumento do valor do pH (Venkatachalam et al., 1992; Ben Mansour et al., 2007; Montes-Atenas et al., 2010). Segundo Venkatachalam et al. (1992), em meio alcalino as bolhas de hidrogênio são de aproximadamente $20 \mu \mathrm{m}$, enquanto que as bolhas de oxigênio podem ser de até $200 \mu \mathrm{m}$. 
Em seu estudo, Chen (2004) mostrou que a densidade da corrente, o tipo de eletrodo e o pH da polpa interferem no diâmetro das bolhas geradas por eletroflotação. Como pode ser visto na Tabela 3, as menores bolhas de hidrogênio foram geradas em $\mathrm{pH}$ neutro. Por outro lado, as menores bolhas de oxigênio foram produzidas em meios ácidos.

No estudo realizado por Bhaskar Raju e Khangaonkar (1984), o tamanho das bolhas de oxigênio formadas no eletrodo de platina foi estimado entre 10 e $45 \mu \mathrm{m}$.

Tabela 3. Tamanho de bolhas em diferentes valores de $\mathrm{pH}$ e eletrodos (adaptada de Chen, 2004).

\begin{tabular}{c|c|c|c|c}
\hline \multirow{2}{*}{$\mathrm{pH}$} & \multicolumn{3}{|c|}{ Bolhas de Hidrogênio $(\mu \mathrm{m})$} & Bolhas de Oxigênio $(\mu \mathrm{m})$ \\
\cline { 2 - 5 } & \multicolumn{3}{|c}{ Material do catodo } & Material do anodo \\
\cline { 2 - 5 } & Platina & Aço & Carbono & Platina \\
\hline 2 & $45-90$ & $20-80$ & $18-60$ & $15-30$ \\
\hline 7 & $5-30$ & $5-45$ & $5-80$ & $17-50$ \\
\hline 12 & $17-45$ & $17-60$ & $17-60$ & $30-70$ \\
\hline
\end{tabular}

\section{RECUPERAÇÃO DE PARTÍCULAS MINERAIS FINAS POR ELETROFLOTAÇÃO}

A aplicação da eletroflotação para a recuperação de finos de minérios tem sido objeto de investigação desde 1946, quando foi inicialmente aplicada no beneficiamento mineral na antiga União Soviética. Recentemente novas pesquisas têm sido realizadas devido ao aumento da demanda de minérios e das baixas eficiências das técnicas convencionais de flotação para o processamento de finos e ultrafinos de minérios (Miettinem et al., 2010).

Bhaskar Raju e Khangaonkar (1982) estudaram a eletroflotação de partículas ultrafinas de calcopirita $(\sim 4 \mu \mathrm{m})$ em um tubo de Hallimond modificado, utilizando etilxantato de potássio (coletor), sulfato de sódio (eletrólito suporte) e dois sistemas de eletrodos: platina(anodo)-cobre(catodo) e grafite(anodo)-cobre(catodo). Neste estudo foram variados os tipos de bolhas geradas eletroliticamente, oxigênio ou hidrogênio, a densidade da corrente, o $\mathrm{pH}$ da solução e a concentração do coletor. A Figura 2 apresenta os principais resultados dos estudos realizados por Bhaskar Raju e Khangaonkar (1982). Os autores concluíram que as pequenas bolhas geradas na eletroflotação são eficientes para a recuperação de partículas ultrafinas de calcopirita. As bolhas de oxigênio geradas a partir do processo eletrolítico promoveram uma maior flotabilidade destes ultrafinos, resultado de uma adesão bolhapartícula mais rápida e eficiente. 


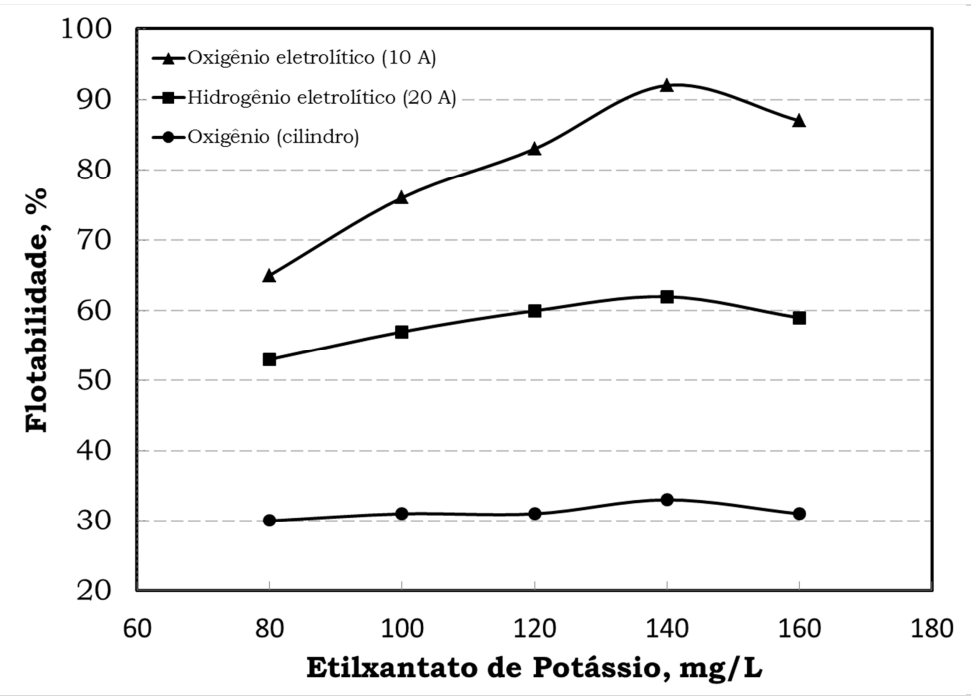

Condições experimentais: coletor [etilxantato de potássio $]=140 \mathrm{mg} \cdot \mathrm{L}^{-1} ; \mathrm{pH}=8$; condicionamento $=2 \mathrm{~min}$; flotação $=3 \mathrm{~min}$; eletrólito suporte [sulfato de sódio] = 0,2817 M; eletrodos: cobre (catodo) e platina (anodo); $\mathrm{D}_{\text {médio }}=\sim 4 \mu \mathrm{m} ;$ Fluxo de gás(cilindro) $=0,5$ L. $\mathrm{min}^{-1}$

Figura 2. Flotabilidade dos ultrafinos de calcopirita nas melhores condições em diferentes sistemas (adaptada de Bhaskar Raju e Khangaonkar, 1982).

Bhaskar Raju e Khangaonkar (1984) estudaram a eletroflotação de ultrafinos de calcopirita $(\sim 4 \mu \mathrm{m})$ em tubo de Hallimond modificado, utilizando eletrodos de platina e de cobre (catodos) e de platina (anodo). Neste estudo variou-se a densidade da corrente, o pH da solução, a concentração do coletor (dietilditiofosfato de sódio), bem como o tipo de bolhas, geradas eletroliticamente (oxigênio ou hidrogênio) e a partir de cilindro de oxigênio puro. A Tabela 4 apresenta os principais resultados dos estudos realizados por Bhaskar Raju e Khangaonkar (1984). Observa-se que a utilização de bolhas geradas eletroliticamente apresentaram flotabilidades superiores ( 81,3\%), quando comparadas aos estudos com bolhas geradas a partir de cilindro de oxigênio ( 39,1\%).

Tabela 4. Flotabilidade dos ultrafinos de calcopirita nas melhores condições em diferentes sistemas (adaptada de Bhaskar Raju e Khangaonkar, 1984).

\begin{tabular}{|c|c|c|c|c|}
\hline \multirow[b]{2}{*}{ Sistema } & \multirow{2}{*}{$\begin{array}{c}\text { Densidade de } \\
\text { corrente } \\
\left(\mathrm{mA} \cdot \mathrm{cm}^{-2}\right)\end{array}$} & \multicolumn{3}{|c|}{ Flotabilidade (\%) } \\
\hline & & $\begin{array}{c}\text { Bolhas de } \mathrm{H}_{2} \\
\text { eletrolítico }\end{array}$ & $\begin{array}{c}\text { Bolhas de } \mathrm{O}_{2} \\
\text { eletrolítico }\end{array}$ & $\begin{array}{c}\text { Bolhas de } \mathrm{O}_{2} \\
\text { (cilindro)* }\end{array}$ \\
\hline Sem coletor & 111 & 48,14 & 63,75 & - \\
\hline \multirow{3}{*}{ Com coletor } & - & - & - & 39,10 \\
\hline & 111 & - & 81,30 & - \\
\hline & 147 & 81,32 & - & - \\
\hline
\end{tabular}

Condições experimentais: coletor [dietilditiofosfato de sódio] = 5,326.10 ${ }^{-4} \mathrm{M} ; \mathrm{pH}=9$; condicionamento $=2 \mathrm{~min}$; eletrodos: cobre (catodo) e platina (anodo); tamanho médio de partícula $=\sim 4 \mu \mathrm{m}$.

* fluxo de gás(cilindro) $=0,5$ L.min ${ }^{-1}$. 
Ketkar et al. (1991) estudaram a eletroflotação de partículas finas e ultrafinas de quartzo em um tubo de Hallimond modificado. Os reagentes utilizados foram o sulfato de cobre (ativador), oleato de sódio (coletor) e sulfato de sódio (eletrólito). Neste estudo foram avaliadas a influência dos tamanhos das bolhas de oxigênio e hidrogênio, geradas eletroliticamente, a densidade da corrente (125 a 325 A.m $\left.{ }^{-2}\right), p H(7$ a 10,5), concentração do coletor $\left(0,2\right.$ a 2,5 mg. $\left.\mathrm{L}^{-1}\right)$, percentagem de sólidos da polpa (0,5 a 5\%), tipo de eletrodo (aço inoxidável - catodo - e a platina - anodo - em diferentes malhas) e o tamanho das amostras de quartzo puro $(-30+20 \mu \mathrm{m},-20+10 \mu \mathrm{m}$ e $-10+4 \mu \mathrm{m})$ obtidas por decantação. Os estudos com a amostra de quartzo fino $(-30+20 \mu \mathrm{m})$ mostraram que é possível se obter uma recuperação de $94 \%$, utilizando bolhas de hidrogênio, para uma concentração do coletor de $2,5 \mathrm{mg} \cdot \mathrm{L}^{-1}$, em $\mathrm{pH} 9,0$, percentagem de sólidos de $0,5 \%$ e densidade da corrente de $250 \mathrm{~A} . \mathrm{cm}^{-2}$. Os autores concluíram que a recuperação de quartzo fino cresce com o aumento da densidade de corrente em todas as frações de tamanho testadas. Dependendo do tamanho de partículas utilizado é necessária a utilização de eletrodos específicos. De acordo com os autores, os melhores resultados para a fração de partículas finas $(-10+4 \mu \mathrm{m})$ foram obtidos com placa de aço inoxidável (catodo), enquanto para frações de tamanho intermediarias $(-20+10 \mu \mathrm{m})$ as melhores recuperações foram obtidas com malha do catodo de 200 mesh. Já para as frações de tamanho maior $(-30+20 \mu \mathrm{m})$, as melhores flotabilidades foram obtidas com malha do catodo de 100 mesh, utilizando elevada densidade de corrente $\left(375 \mathrm{~A} \cdot \mathrm{cm}^{-2}\right)$.

Llerena et al. (1996) estudaram a eletroflotação de partículas finas de esfalerita $(<25 \mu \mathrm{m})$ em uma célula de bancada adaptada com um par de eletrodos de aço inoxidável 304 (115 $\mu \mathrm{m}$ de diâmetro). Os reagentes utilizados foram o sulfato de sódio pentahidratado (123 mg. $\mathrm{L}^{-1}$ - ativador), sulfato de sódio $(0,12 \mathrm{M}$ - eletrólito suporte), Aero 343 - xantato isopropílico de sódio (192 mg. L $^{-1}$ - coletor) e hidróxido de sódio e ácido sulfúrico (reguladores de $\mathrm{pH}$ ). A densidade da corrente utilizada foi $50 \mathrm{~mA} \cdot \mathrm{cm}^{-2}$. No estudo avaliou-se a influência da relação gases gerados pela eletrólise $\left(\mathrm{H}_{2}\right.$ e/ou $\left.\mathrm{O}_{2}\right)-\mathrm{pH}$, na recuperação dos finos de esfalerita. Os resultados mostraram que com a utilização de bolhas de hidrogênio foi possível a obtenção de recuperações próximas a 100\% dos finos de esfalerita na faixa de $\mathrm{pH}$ entre 2,5 e 4,0 para um tempo de flotação de aproximadamente 4 minutos. Os estudos realizados com bolhas de oxigênio apresentaram resultados semelhantes aos obtidos com bolhas de hidrogênio, isto é, recuperação de $100 \%$ dos finos de esfalerita em pH 4 . Os autores compararam estes resultados com os do estudo da flotabilidade, da mesma amostra, em célula de bancada convencional utilizando bolhas geradas a partir de difusores, nas mesmas condições, entretanto obtiveram recuperações inferiores. Os autores concluíram que esta diferença pode ser atribuída provavelmente pela diferença dos tamanhos das bolhas geradas nos dois processos. As bolhas geradas no processo convencional a partir de difusores $(200 \mu \mathrm{m})$ são bem maiores do que as bolhas geradas no processo eletrolítico (16 a $31 \mu \mathrm{m}$ ). Porém, de acordo com os autores, a eletroflotação dos finos de esfalerita nas melhores condições encontradas, necessitam de uma elevada quantidade de energia (65 $\mathrm{kWh} . \mathrm{m}^{-3}$ ) quando comparadas com a flotação convencional $\left(3 \mathrm{kWh} \cdot \mathrm{m}^{-3}\right)$.

Sarkar et al. (2010) estudaram a eletroflotação de ultrafinos de sílica ( $13 \mu \mathrm{m}$ ) em célula de flotação (Denver) modificada, utilizando eletrodos de aço inoxidável (catodo) e de carbono (anodo). Os autores compararam a flotação com bolhas de hidrogênio geradas 
eletroliticamente, com as bolhas geradas por difusores na flotação convencional. Neste

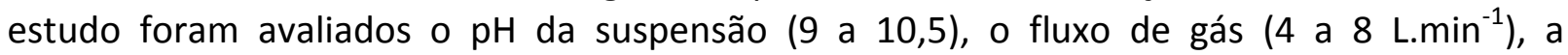
concentração do Brometo de Cetil Trimetil Amônio - CTAB $\left(4,4 \times 10^{-6}\right.$ a $27,4 \times 10^{-6} \mathrm{M}$ - coletor), do Metil Isobutil Carbinol - MIBC (29 ppm - espumante) e do sulfato de sódio (0,005 a 0,1 M eletrólito suporte). Foram mantidos constantes a densidade da corrente, a percentagem de sólidos (2\%), o tamanho de partícula e a velocidade de agitação. As melhores recuperações ( 75\%) dos ultrafinos de sílica na flotação convencional utilizando bolhas geradas por difusores foram obtidas na faixa de $\mathrm{pH} 9,5$ a 10,0. Os estudos também mostraram que as recuperações destes ultrafinos, para ambos os tipos de geração de bolhas, aumentaram com a elevação do fluxo dos gases e da concentração do coletor, enquanto que o aumento da concentração do sulfato de sódio promoveu uma diminuiu da recuperação da sílica em ambos os sistemas. Os autores mostraram que com a utilização das bolhas de hidrogênio no processo de flotação, foi possível obter recuperações dos ultrafinos de sílica da ordem de $95 \%$.

\section{CONCLUSÃO}

Conforme mostra a literatura descrita no presente relato, a eletroflotação apresentase como uma alternativa para o tratamento de partículas minerais finas e ultrafinas, principalmente pelo fato de gerar bolhas menores que as bolhas utilizadas nos processos de flotação convencional, aumentando assim a eficiência de colisão entre as bolhas e as partículas. Entretanto, novos estudos devem ser realizados para comprovar a eficácia da técnica, bem como avaliar o consumo energético desta alternativa, que requer uma condutividade elétrica adequada da polpa para viabilizar o processo.

\section{REFERÊNCIAS BIBLIOGRÁFICAS}

1. BEN MANSOUR, L., KSENTINI, I., ELLEUCH, B. Treatment of wastewaters of paper industry by coagulation-electroflotation. Desalination, v.208, n.1-3, p.34-41, 2007.

2. BHASKAR RAJU, G., KHANGAONKAR, P.R. Electroflotation of chalcopyrite fines. International Journal of Mineral Processing, v.9, n.2, p.133-143, 1982.

3. BHASKAR RAJU, G., KHANGAONKAR, P.R. Electroflotation of chalcopyrite fines with sodium diethyldithiocarbamate as collector. International Journal of Mineral Processing, v.13, n.3, p.211-221, 1984.

4. BURNS, S. E., YIACOUMI, S., TSOURIS, C. Microbubble generation for environmental and industrial separations. Separation and Purification Technology, v.11, n.3, p.221-232, 1997.

5. CHEN ,G. Electrochemical technologies in wastewater treatment. Separation and Purification Technology, v.38, n.1, p.11-41, 2004.

6. COLLINS, D. N., READ, A. D. The treatment of slimes. Mineral Science and Engineering, v.3, p.19-31, 1971. 
7. GLEMBOTSKII, V.A., MAMAKOV, A.A., SOROKINA, V.N. Size of gas bubbles formed under electroflotation conditions. Elektronnaya Obrabotka Materialov, v.5, p.66-68, 1973.

8. INAN, H., ANATOLY DIMOGLO, SIMSEK, H., KARPUZCU, M. Olive oil mill wastewater treatment by means of electro-coagulation. Separation and Purification Technology, v.36, n.1, p.23-31, 2004.

9. JANSSEN, L.J.J., HOOGLAND, J.G. The effect of electrolytically evolved gas bubbles on the thickness of the diffusion layer. Electrochimica Acta, v.15, p.1013-1023, 1970.

10. KETKAR, D.R., MALLIKARJUNAN, R., VENKATACHALAM, S. Electroflotation of quartz fines. International Journal of Mineral Processing, v.31, n.1-2, p.127-138, 1991.

11. LANDOLT, D., ACOSTA, R., MULLER, R.H., TOBAIS, C.W. An optical study of cathodic hydrogen evolution in high rate electrolysis. Journal of the Electrochemical Society, v.117, n.6, p.839-845, 1970.

12. LLERENA, C., HO, J.C.K., PIRON, D.L. Effect of pH on electroflotation of sphalerite. Chemical Engineering Communication, v.155, p.217-228, 1996.

13. MIETTINEN, T., RALSTON, J., FORNASIERO, D. The limits of fine particle flotation. Minerals Engineering, v.23, n.5, p.420-437, 2010.

14. MONTES-ATENAS, G., GARCIA-GARCIA, F.J., MERMILLOD-BLONDIN, R., MONTES, S., Effect of suspension chemistry onto voltage drop: Application to electro-flotation. Powder Technology, v.204, n.1, p.1-10, 2010.

15. NGUYEN, A.V., GEORGE, P., JAMESON, G.J. Demonstration of a minimum in the recovery of nanoparticles by flotation: theory and experiment. Chemical Engineering Science, v.61, n.8, p.2494-2509, 2006.

16. RUBio, J., CAPPONI, F., MATIOLO, E., NUNES, D., GUERRERO, C. P., BERKOWITZ, G. Advances in flotation of mineral fines. In: Proceedings XXII International Mineral Processing Congress, p.1014-1022, 2003.

17. RUBIO, J., CAPPONI, F., MATIOLO, E., ROSA, J. J. Avanços na flotação de finos de minérios sulfetados de cobre e molibdênio. In: XXI Encontro Nacional de Tratamento de Minérios e Metalurgia Extrativa, Florianópolis-SC, V.2, p.69-78, 2004.

18. SARKAR, M.S.K.A., DONNE, S.W., EVANS, G.M. Hydrogen bubble flotation of silica. Advanced Powder Technology, v.21, n.4, p.412-418, 2010.

19. SARROT, V., HUANG, Z., LEGENDRE, D., GUIRAUD, P. Experimental determination of particles capture efficiency in flotation. Chemical Engineering Science, v.62, n.24, p. 73597369, 2007.

20. SIVAMOHAN, R., The problem of recovering very fine particles in mineral processing - A review. International Journal of Mineral Processing, v.28, p.247-288, 1990.

21. SONG, S., LOPEZ-VALDIESO, A., REYES-BAHENA, J. L.,LARA-VALENZUELA, C., Floc flotation of galena and sphalerite fines. Minerals Engineering, v.14, n.1, p.87-98, 2001. 
22. SUBRAHMANYAM, T. V., FORSSBERG, E.F.S. Fine particle processing: Shear flocculation and carrier flotation - A review. International Journal of Mineral Processing, v.30, n.3-4, p.265286, 1990.

23. TRAHAR, W. J. A rational interpretation of role of particle size inflotation. International Journal of Mineral Processing, v.2, p.289-327, 1981.

24. TRAHAR, W. J., 1981. A rational interpretation of role of particle size in flotation. International Journal of Mineral Processing, v.2, p.289-327.

25. TRAHAR, W. J., WARREN, L. J. The flotability of very fine particles - A review. International Journal of Mineral Processing, v.3, n.2, p.103-131, 1976.

26. VENKATACHALAM, S. Electrogenerated Gas Bubbles in Flotation. Mineral Processing and Extractive Metallurgy Review, v.8, p.47-55, 1992.

27. WARREN, L. J. Determination of the contributions of true flotation and entrainment in batch flotation tests. International Journal of Mineral Processing, v.14, n.1, p.33-44, 1985.

28. WALSH, F.C., HERRON, M.H., Electrocrystallization and electrochemical control of crystal growth: fundamental considerations and electrodeposition of metals. Journal of Physics D: Applied Physics, v.24, n.2, p.217-229, 1991.

29. YOON, R.H. Microbubble flotation. Minerals Engineering, v.6 n.6, p.619-630, 1993. 